\title{
Spatial corrections of ROSAT HRI observations
}

\author{
D.E. Harris ${ }^{1}$, J.D. Silverman ${ }^{1}$, G. Hasinger ${ }^{2}$, and I. Lehmann ${ }^{2}$ \\ 1 Harvard-Smithsonian Center for Astrophysics, 60 Garden Street, Cambridge, MA 02138, U.S.A. \\ 2 Astrophysikalisches Institut Potsdam, An der Sternwarte 16, D-14482 Potsdam, Germany
}

Received June 29; accepted July 7, 1998

\begin{abstract}
X-ray observations with the ROSAT High Resolution Imager (HRI) often have spatial smearing on the order of $10^{\prime \prime}$ (Morse 1994). This degradation of the intrinsic resolution of the instrument $\left(5^{\prime \prime}\right)$ can be attributed to errors in the aspect solution associated with the wobble of the space craft or with the reacquisition of the guide stars. We have developed a set of IRAF/PROS and MIDAS/EXSAS routines to minimize these effects. Our procedure attempts to isolate aspect errors that are repeated through each cycle of the wobble. The method assigns a "wobble phase" to each event based on the 402 second period of the ROSAT wobble. The observation is grouped into a number of phase bins and a centroid is calculated for each sub-image. The corrected HRI event list is reconstructed by adding the sub-images which have been shifted to a common source position. This method has shown $\sim 30 \%$ reduction of the full width half maximum $(F W H M)$ of an X-ray observation of the radio galaxy 3C 120. Additional examples are presented.
\end{abstract}

Key words: techniques: image processing - X-rays: general

\section{Introduction}

Spatial analysis of ROSAT HRI observations is often plagued by poor aspect solutions, precluding the attainment of the potential resolution of about $5^{\prime \prime}$. In many cases (but not all), the major contributions to the degradation in the effective Point Response Function (PRF) come from aspect errors associated either with the ROSAT wobble or with the reacquisition of the guide stars.

To avoid the possibility of blocking sources by the window support structures (Positional Sensitive Proportional Counter) or to minimize the chance that the pores near the center of the microchannel plate would become burned out

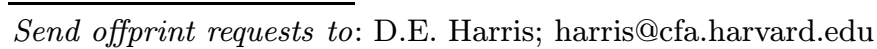

from excessive use (High Resolution Imager), the satellite normally operates with a constant dither for pointed observations. The period of the dither is $402 \mathrm{~s}$ and the phase is tied to the spacecraft clock. Any given point on the sky will track back and forth on the detector, tracing out a line of length $\approx 3$ arcmin with position angle of $135^{\circ}$ in raw detector coordinates (for the HRI). Imperfections in the star tracker (see Sect. 2) can produce an erroneous image if the aspect solution is a function of the wobble track on the CCD of the star tracker.

This work is similar to an analysis by Morse (1994) except that we do not rely on a direct correlation between spatial detector coordinates and phase of the wobble. Moreover, our method addresses the reacquisition problem which produces the so-called cases of "displaced OBIs". An "OBI" is an observation interval, normally lasting for $1 \mathrm{ks}$ to $2 \mathrm{ks}$ (i.e. a portion of an orbit of the satellite). A new acquisition of the guide stars occurs at the beginning of each OBI and we have found that different aspect solutions often result. Occasionally a multi-OBI observation consists of two discrete aspect solutions. A recent example (see Sect. 7.1.2) showed one OBI for which the source was $10^{\prime \prime}$ north of its position in the other 17 OBIs. Note that this sort of error is quite distinct from the wobble error.

Throughout this discussion, we use the term "PRF" in the dynamic sense: it is the point response function realized in any given situation: i.e. that which includes whatever aspect errors are present. We start with an observation for which the PRF is much worse than it should be. We seek to improve the PRF by isolating the offending contributions and correcting them if possible or rejecting them if necessary.

\section{Model and method}

The "model" for the wobble error assumes that the star tracker's CCD has some pixels with different gain than others. As the wobble moves the de-focused star image across the CCD, the centroiding of the stellar image gets the wrong value because it is based on the relative 
response from several pixels. If the roll angle is stable, it is likely that the error is repeated during each cycle of the wobble since the star's path is over the same pixels (to a first approximation if the aspect "jitter" is small compared to the pixel size of $\approx 1$ arcmin). What is not addressed is the error in roll angle induced by erroneous star positions. If this error is significant, the centroiding technique with one strong source will fix only that source and its immediate environs.

The correction method assigns a "wobble phase" to each event; then divides each OBI (or other suitably defined time interval) into a number of wobble phase bins. The centroid of the reference source is measured for each phase bin. The data are then recombined after applying $x$ and $y$ offsets in order to ensure that the reference source is aligned for each phase bin. What is required is that there are enough counts in the reference source to obtain a reliable centroid. Variations of this method for sources weaker than approx 0.1 count/s involve using all OBIs together before dividing into phase bins. This is a valid approach so long as the nominal roll angle is stable (i.e. within a few tenths of a degree) for all OBIs, and so long as major shifts in the aspect solutions of different OBIs are not present.

\section{Diagnostics}

Our normal procedure for evaluation is to measure the $F W H M$ (both the major and minor axes) of the observed response on a map smoothed with a $3^{\prime \prime}$ Gaussian. For the best data, we find the resulting $F W H M$ is close to $5.7^{\prime \prime}$. While there are many measures of source smearing, we prefer this approach over measuring radial profiles because there is no uncertainty relating to the position of the source center; we are normally dealing with elliptical rather than circular distributions; and visual inspection of the two dimensional image serves as a check on severe abnormalities. It has been our experience that when we are able to reduce the $F W H M$ of the PRF, the wings of the PRF are also reduced.

\subsection{Wobble errors}

If the effective PRF is evaluated for each OBI separately, the wobble problem is manifest by a degraded PRF in one or more OBIs. Most OBIs contain only the initial acquisition of the guide stars, so when the PRF of a particular OBI is smeared, it is likely to be caused by the wobble error and the solution is to perform the phased "de-wobbling".

\subsection{Misplaced $O B I$}

For those cases where each OBI has a relatively good PRF but the positions of each centroid have significant disper- sion, the error cannot be attributed to the wobble. We use the term "misplaced OBI" to describe the situation in which a different aspect solution is found when the guide stars are reacquired. In the worst case, multiple aspect solutions can produce an image in which every source in the field has a companion displaced by anywhere from 10 to 30 arcsec or more. When the separation is less than 10 arcsec, the source can appear to have a tear drop shape (see Sect. 7.1.1) or an egg shape. However, depending on the number of different aspect solutions, almost any arbitrary distortion to the (circularly symmetric) ideal PRF is possible. The fix for these cases is simply to find the centroid for each OBI, and shift them before co-adding (e.g., see Morse et al. 1995).

\section{IRAF/PROS implementation}

The ROSAT Science Data Center (RSDC) at SAO has developed scripts to assist users in evaluating individual OBIs and performing the operations required for dewobbling and alignment. The scripts are available from our anonftp area: sao-ftp.harvard.edu. Then change directory to pub/rosat/dewob.

An initial analysis needs to be performed to determine the stable roll angle intervals, to check for any misalignment of OBIs and to examine the guide star combinations. These factors together with the source intensity are important in deciding what can be done and the best method to use.

\subsection{OBI by $O B I$ method}

If the observation contains a strong source $(\geq 0.1$ counts/s) near the field center (i.e. close enough to the center that the mirror blurring is not important), then the preferred method is to dewobble each OBI. The data are thus divided into $n \times p$ qpoe files ( $n=$ number of OBIs; $p=$ number of phase bins). The position of the centroid of the reference source is determined and each file is shifted in $x$ and $y$ so as to align the centroids from all OBIs and all phase bins. The data are then co-added or stacked to realize the final image (qpoe file).

\subsection{Stable roll angle intervals}

For sources weaker than 0.1 counts/s, it is normally the case that there are not enough counts for centroiding when 10 phase bins are used. If it is determined that there are no noticeable shifts between OBIs, then it is possible to use many OBIs together so long as the roll angle does not change by a degree or more. 


\subsection{Method for visual inspection}

On rare occasions, it may be useful to examine each phase bin visually to evaluate the segments in order to decide if some should be deleted before restacking for the final result. We have found it useful to do this via contour diagrams of the source. This approach can be labor intensive if there are a large number of OBIs and phase bins but scripts we provide do most of the manipulations.

\section{MIDAS/EXSAS implementation}

The X-ray group at the Astrophysical Institute Potsdam (AIP) has developed some MIDAS/EXSAS routines to correct for the ROSAT wobble effect. The routines can be obtained by anonymous ftp from ftp.aip.de at directory pub/users/rra/wobble. The correction procedure works interactively in five main steps:

- Choosing of a constant roll angle interval.

- Folding the data over the $402 \mathrm{~s}$ wobble period.

- Creation of images using 5 or 10 phase intervals.

- Determining the centroid for the phase resolved images.

- Shifting the photon $X / Y$ positions in the events table.

We have tested the wobble correction procedures for 21 stars and 24 galaxies of the ROSAT Bright Survey using archival HRI data. The procedures work successfully down to an HRI source count rate of about 0.1 counts/s. In the case of lower count rates the determination of the centroid position failed because of the few photons available in the phase-binned images. The number of phase bins which can be used is of course dependent on the X-ray brightness of the source.

\section{Limitations}

We briefly describe the effects which limit the general use of the method. In so doing, we also indicate the process one can use in deciding if there is a problem, and estimating the chances of substantial improvement.

\subsection{Presence of aspect smearing}

The $F W H M$ of all sources in the field should be $\geq 7^{\prime \prime}$ (after smoothing with a $3^{\prime \prime}$ Gaussian). If any source is smaller than this value, it is likely that aspect problems are minimal and little is to be gained by applying the dewobbling method.

If there is only a single source in the field, without a priori knowledge or further analysis it is difficult to determine whether a distribution significantly larger than the ideal PRF is caused by source structure or aspect smearing. The best approach in this case is to examine
Table 1. ROSAT clock resets

\begin{tabular}{cl}
\hline & \\
Year & Day \\
\hline 90 & 151.87975 (launch) \\
91 & 25.386331 \\
92 & 42.353305 \\
93 & 18.705978 \\
94 & 19.631352 \\
95 & 18.169322 \\
96 & 28.489871 \\
97 & 16.069990 \\
98 & 19.445738 \\
\hline
\end{tabular}

the image for each OBI separately to see if some or all are smaller than the total image (i.e. OBI aspect solutions are different).

\subsection{Wobble phase}

It is important that the phase of the wobble is maintained. This is ensured if there is no "reset" of the space craft clock during an observation. If an observation has a begin and end time/date that includes a reset, it will be necessary to divide the data into two segments with a time filter before proceeding to the main analysis. Dates of clock resets (Table 1) are provided by MPE: http://www.ROSAT.mpegarching.mpg.de/ prp/timcor.html.

\subsection{Characteristics of the reference source}

In most cases, the reference source (i.e. the source used for centroiding) will be the same as the target source, but this is not required. Ideally, the reference source should be unresolved in the absence of aspect errors and it should not be embedded in high brightness diffuse emission (e.g. the core of M 87 does not work because of the bright emission from the Virgo Cluster gas). Both of these considerations are important for the operation of the centroiding algorithm, but neither is an absolute imperative. For accurate centroiding, the reference source needs to stand well above any extended component.

Obviously the prime concern is that there be enough counts in a phase bin to successfully measure the centroid. The last item is usually the determining factor, and as a rule of thumb, it is possible to use 10 phase bins on a source of 0.1 counts $/ \mathrm{s}$. We have tested a strong source to see the effect of increasing the number of phase bins. In Fig. 1, we show the results of several runs on an observation of HZ 43 (12 counts/s). This figure demonstrates that ten phase bins is a reasonable choice, but that there is little to be gained by using more than 20 phase bins. 


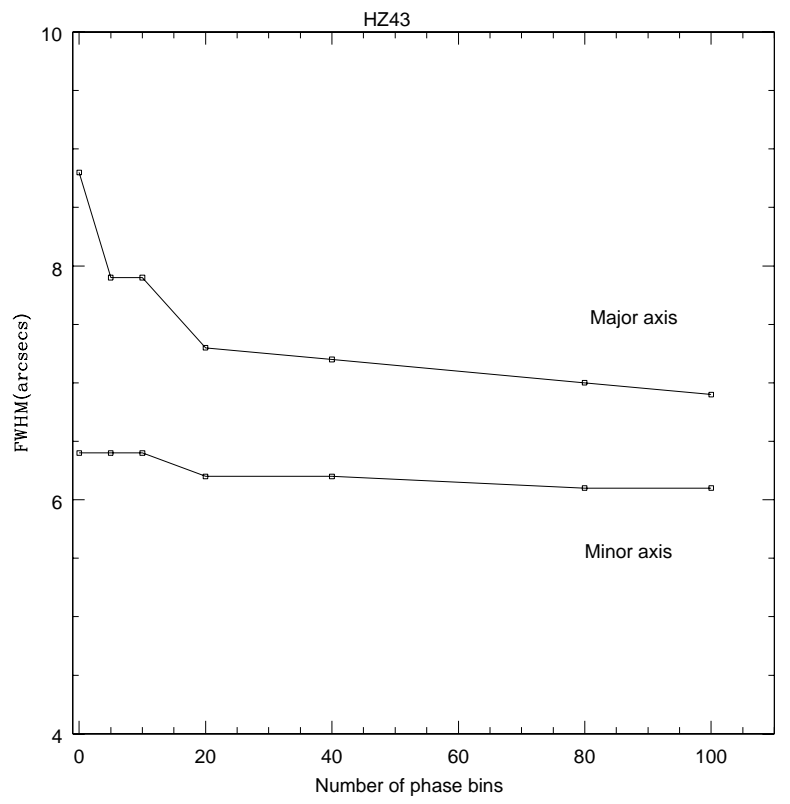

Fig. 1. The $F W H M$ of a HZ43 (observation number rh142545) observation was measured for multiple dewobble runs while increasing the number of phase bins

\section{$3 \mathrm{C} 120$}

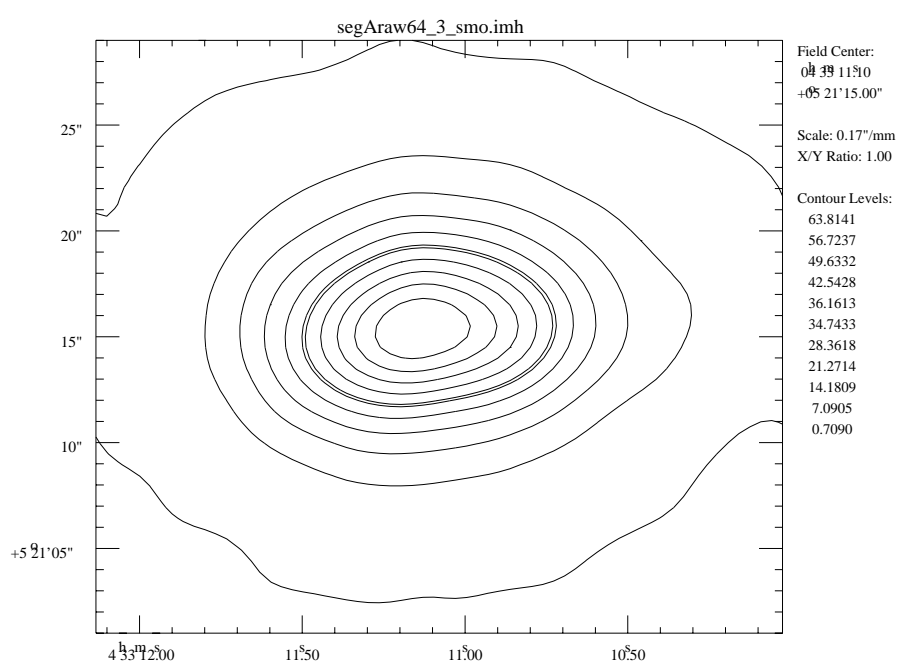

Fig. 2. The original data for 3C 120 (segment A, rh702080n00), smoothed with a Gaussian of $F W H M=3^{\prime \prime}$. The peak value on the map is 70.9 counts per $0.5^{\prime \prime}$ pixel. Contour levels are $1,10,20,30, \ldots 90 \%$ of the peak value, with the $50 \%$ contour, doubled. The nominal roll angle is $-167^{\circ}$ and the wobble direction is at $\mathrm{PA}=122^{\circ}$. The $F W H M$ of this smoothed image is $11.6^{\prime \prime} \times 7.4^{\prime \prime}$

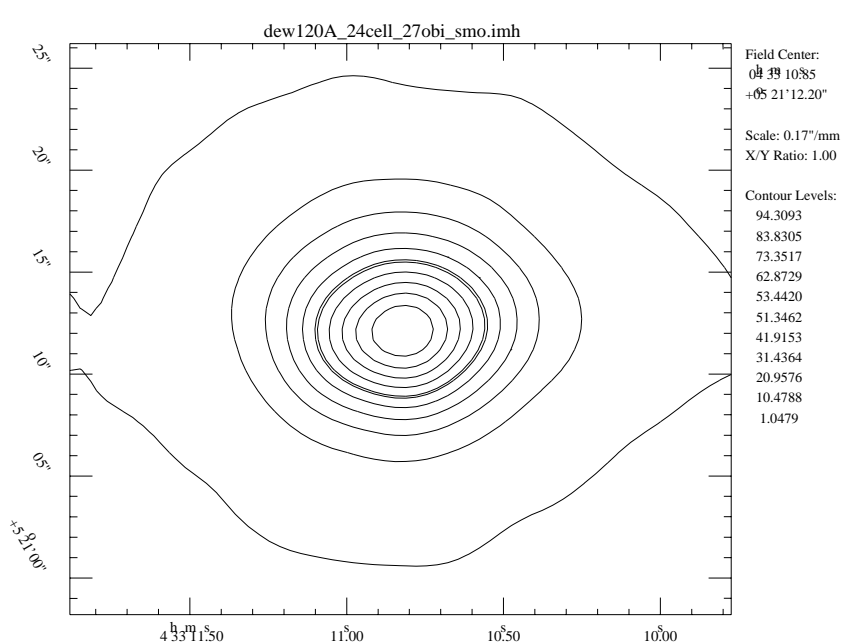

Fig. 3. The results after dewobbling $3 \mathrm{C} 120 \mathrm{~A}$, smoothed with a Gaussian of $F W H M=3^{\prime \prime}$. The peak value on the map is now 104.8 counts per $0.5^{\prime \prime}$ pixel. Contour levels are 1, 10, 20, $30, \ldots 90 \%$ of the peak value, with the $50 \%$ contour, doubled. The FWHM of this smoothed image is $8.1^{\prime \prime} \times 6.7^{\prime \prime}$

\section{Examples}

\section{1. $3 C 120$}

$3 \mathrm{C} 120$ is a nearby radio galaxy $(z=0.033)$ with a prominent radio jet leaving the core at $\mathrm{PA} \approx 270^{\circ}$. The ROSAT HRI observation was obtained in two segments, each of which had aspect problems. Since the average source count rate is 0.8 count/s, the X-ray emission is known to be highly variable (and therefore most of its flux must be unresolved), and each segment consisted of many OBIs, we used these observations for testing the dewobbling scripts.

7.1.1. Segment A: Two aspect solutions, both found multiple times

The smoothed data (Fig. 2) indicated that in addition to the X-ray core, a second component was present, perhaps associated with the bright radio knot $4^{\prime \prime}$ west of the core. When analyzing these two components for variability, it was demonstrated that most of the emission was unresolved, but that the aspect solution had at least two different solutions, and that the change from one to the other usually coincided with OBI boundaries. The guide star configuration table showed that a reacquisition coincided with the change of solution.

The 24 OBIs comprising the $36.5 \mathrm{ksec}$ exposure were obtained between 96Aug16 and 96Sep12. Because 3C 120 is close to the ecliptic, the roll angle hardly changed, and our first attempts at dewobbling divided the data into 2 "stable roll angle intervals". This effort made no noticeable improvement. 


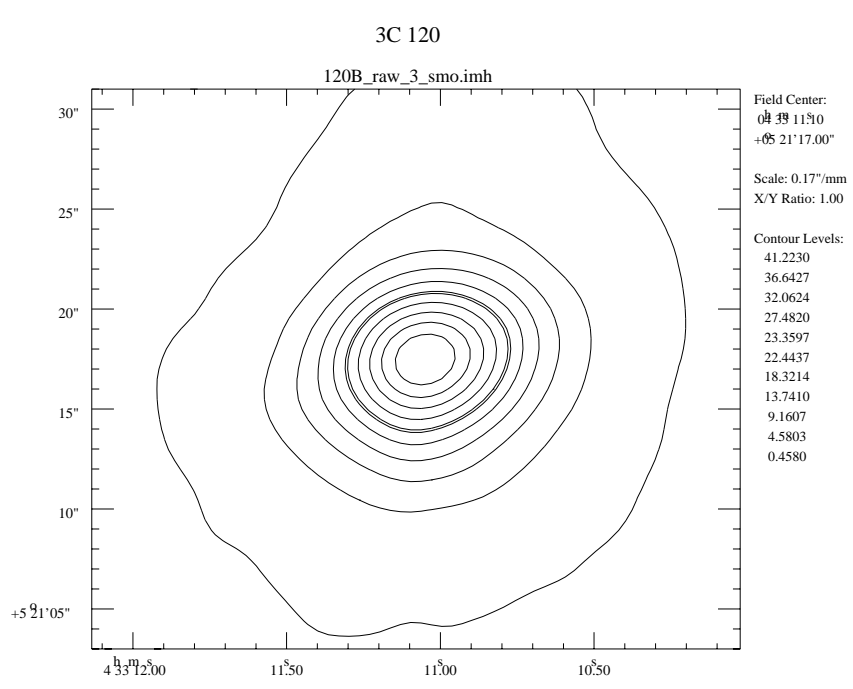

Fig. 4. The original data of 3C 120 (segment B, rh702080a01), smoothed with a Gaussian of $F W H M=3^{\prime \prime}$. The peak value on the map is 45.8 counts per $0.5^{\prime \prime}$ pixel. The contour levels are the same percentage values as those of Fig. 2. The roll angle is $8^{\circ}$ and the wobble $\mathrm{PA}$ is $127^{\circ}$. FWHM for this image is $8.0^{\prime \prime} \times 6.7^{\prime \prime}$

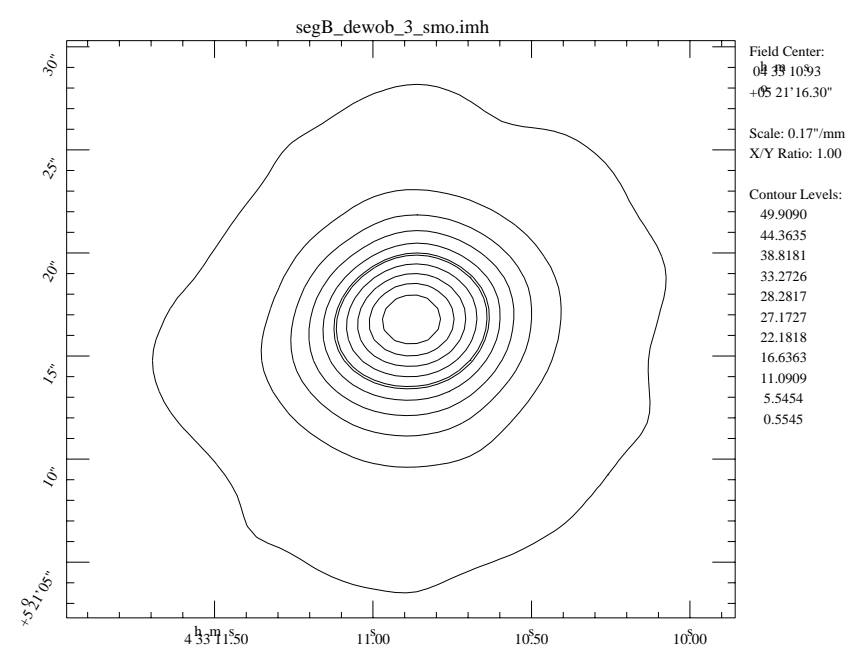

Fig. 5. The results of 3C 120 (segment B) after dewobbling. The contour levels are the same percentage values as those of Fig. 4, but the peak is now 55.4. The FWHM is $7.2^{\prime \prime} \times 6.5^{\prime \prime}$

We then used the method described in Sect. 4.1. The results are shown in Fig. 3. It can be seen that a marked improvement has occurred, but some of the E-W smearing remains.

\subsubsection{Segment B: A single displaced OBI}

The second segment of the 3C 120 observation was obtained in 1997 March. In this case, only one OBI out of

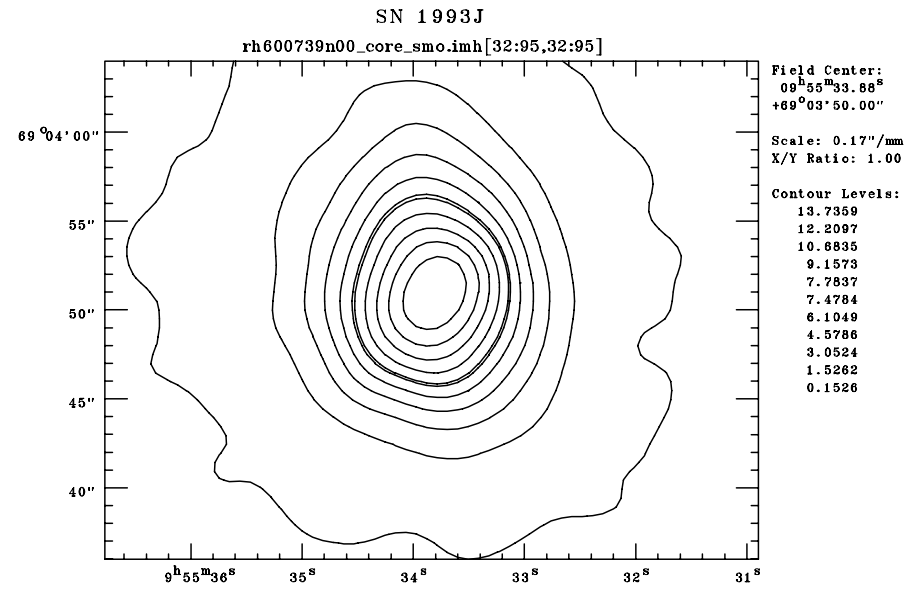

Fig. 6. The original M 81 data (rh600739), smoothed with a Gaussian of $F W H M=3^{\prime \prime}$. The peak value on the map is 15.3 counts per $0.5^{\prime \prime}$ pixel. The contour levels are 1, 10, 20, 30, 40, 50 (the $50 \%$ contour, doubled), 60, 70, 80, and 90 percent of the peak value. The nominal roll angle is $135^{\circ}$ and the wobble direction is $0^{\circ}$. The $F W H M$ of this smoothed image is $10.4^{\prime \prime} \times 7.5^{\prime \prime}$

17 was displaced. It was positioned $10^{\prime \prime}$ to the north of the other positions, producing a low level extension (see Fig. 4). After dewobbling, that feature is gone, the half power size is reduced, and the peak value is larger (Fig. 5).

\section{2. $M 81$}

M 81 is dominated by an unresolved nuclear source. The count rate is 0.31 count/s. The observation has 14 OBIs for a total exposure of 19.9 ks. Figure 6 shows the data from SASS processing. After running the "OBI by OBI" method, the source is more circularly symmetric, has a higher peak value, and a smaller $F W H M$ (Fig. 7).

\section{3. $N G C 5548$}

This source was observed from 25 June to 11 July 1995 for a livetime of $53 \mathrm{ks}$ with $33 \mathrm{OBIs}$. The average count rate was 0.75 counts/s and the original data had a $F W H M=$ $8.2^{\prime \prime} \times 6.8^{\prime \prime}$. Most of the OBIs appeared to have a normal PRF but a few displayed high distortion. After applying the OBI by OBI method, the resulting $F W H M$ was $6.3^{\prime \prime}$ in both directions and the peak value on the smoothed map increased from 138 to 183 counts per $0.5^{\prime \prime}$ pixel.

\subsection{RZ Eri}

The observation of this star was reduced in MIDAS/EXSAS. The source has a count rate of 0.12 count $/ \mathrm{s}$. The reduction selected only a group of 


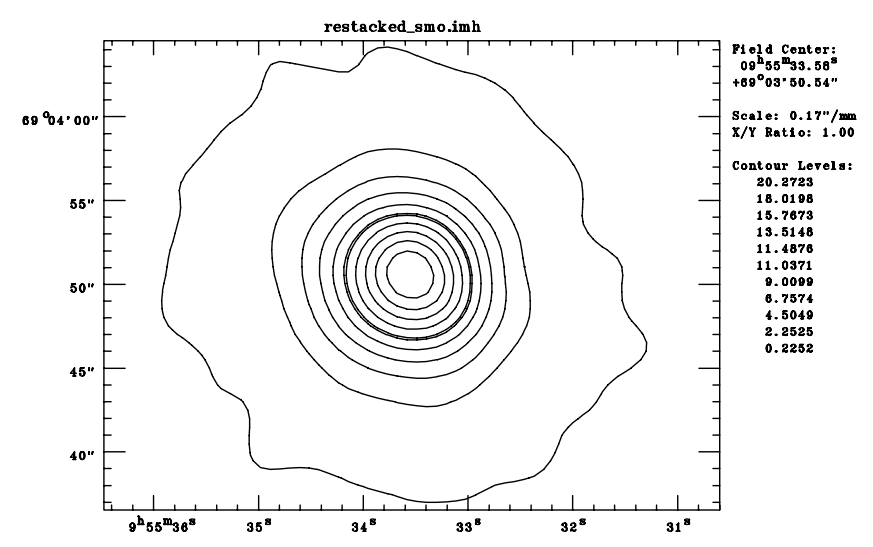

Fig. 7. The results after dewobbling of M 81 smoothed with a Gaussian of $F W H M=3^{\prime \prime}$. The peak value on the map is 22.5 counts per $0.5^{\prime \prime}$ pixel. The contour levels are 1, 10, 20, 30, 40, 50 (the $50 \%$ contour, doubled), 60, 70, 80, and 90 percent of the peak value. Ten phase bins have been used. The FWHM of this smoothed image is $7.2^{\prime \prime} \times 6.5^{\prime \prime}$

the OBIs which comprised a "stable roll angle interval"; almost half the data were rejected. The original smoothed image had a $F W H M=8.4^{\prime \prime} \times 6.6^{\prime \prime}$. After dewobbling, the resulting $F W H M$ was $6.9^{\prime \prime} \times 5.8^{\prime \prime}$.

\section{Summary}

We have developed a method of improving the spatial quality of ROSAT HRI data which suffer from two sorts of aspect problems. This approach requires the presence of a source near the field center which has a count rate of $\approx 0.1$ counts $/ \mathrm{s}$ or greater. Although the method does not fix all bad aspect problems, it produces marked improvements in many cases.

Note added in proof: The "displaced OBI" example of 3C 120 appears to be caused by a bad boresight file since a recent reprocessed image does not have this problem. However, the original data still serve as a valid example.

Acknowledgements. We thank M. Hardcastle (Bristol) for testing early versions of the software and for suggesting useful improvements. J. Morse contributed helpful comments on the manuscript. The 3C 120 data were kindly provided by DEH, A. Sadun, M. Vestergaard, and J. Hjorth (a paper is in preparation). The other data were taken from the ROSAT archives. The work at SAO was supported by NASA contract NAS5-30934.

\section{References}

David L.D., Harnden Jr. F.R., Kearns K.E., Zombeck M.V., 1995, The ROSAT High Resolution Imager (HRI), A hardcopy is available: Center for Astrophysics, RSDC, MS 3, 60 Garden St., Cambridge, MA 02138, U.S.A. (http://heawww.harvard.edu/ROSAT/rsdc_www/hricalrep.html)

Morse J.A., 1994, PASP 106, 675

Morse J.A., Wilson A.S., Elvis M., Weaver K.A., 1995, ApJ 439, 121 